\title{
Multiplicative perturbations of local $C$-cosine functions
}

\author{
Chung-Cheng Kuo and Nai-Sher Yeh
}

\begin{abstract}
We establish some left and right multiplicative perturbations of a local $C$-cosine function $C(\cdot)$ on a complex Banach space $X$ with non-densely defined generator, which can be applied to obtain some new additive perturbation results concerning $C(\cdot)$.
\end{abstract}

Mathematics Subject Classification (2010): 47D60, 47D62.

Keywords: Local $C$-cosine function, subgenerator, generator, abstract Cauchy problem.

\section{Introduction}

Let $X$ be a Banach space over $\mathbb{F}(=\mathbb{R}$ or $\mathbb{C})$ with norm $\|\cdot\|$, and let $L(X)$ denote the set of all bounded linear operators on $X$. For each $0<T_{0} \leq \infty$ and each injection $C \in L(X)$, a family $C(\cdot)\left(=\left\{C(t) \mid 0 \leq t<T_{0}\right\}\right)$ in $L(X)$ is called a local $C$-cosine function on $X$ if it is strongly continuous, $C(0)=C$ on $X$ and satisfies

(1.1) $2 C(t) C(s)=C(t+s) C+C(|t-s|) C$ on $X$ for all $0 \leq t, s, t+s<T_{0}$

(see [7], [10], [14], [20], [22], [24], [26]). In this case, the generator of $C(\cdot)$ is a linear operator $A$ in $X$ defined by

$$
D(A)=\left\{x \in X \mid \lim _{h \rightarrow 0^{+}} 2(C(h) x-C x) / h^{2} \in R(C)\right\}
$$

and $A x=C^{-1} \lim _{h \rightarrow 0^{+}} 2(C(h) x-C x) / h^{2}$ for $x \in D(A)$. Moreover, we say that $C(\cdot)$ is

(1.2) locally Lipschitz continuous, if for each $0<t_{0}<T_{0}$ there exists a $K_{t_{0}}>0$ such that $\|C(t+h)-C(t)\| \leq K_{t_{0}} h$ for all $0 \leq t, h, t+h \leq t_{0}$;

(1.3) exponentially bounded, if $T_{0}=\infty$ and there exist $K, \omega \geq 0$ such that $\|C(t)\| \leq K e^{\omega t}$ for all $t \geq 0$

(1.4) exponentially Lipschitz continuous, if $T_{0}=\infty$ and there exist $K, \omega \geq 0$ such that $\|C(t+h)-C(t)\| \leq K h e^{\omega(t+h)}$ for all $t, h \geq 0$. 
In general, a local $C$-cosine function is also called a $C$-cosine function if $T_{0}=\infty$ (see [17], [6], [4], [13]), a $C$-cosine function may not be exponentially bounded (see [13]), and the generator of a local $C$-cosine function may not be densely defined (see [17], [6]). Moreover, a local $C$-cosine function is not necessarily extendable to the half line $[0, \infty)$ (see [22]) except for $C=I$ (identity operator on $X$ ). Perturbations of local $C$-cosine functions with or without the exponential boundedness have been extensively studied by many authors appearing in $[2,6,8-17,19,23,25]$. Some interesting applications of this topic are also illustrated there. In particular, Li has obtained some right-multiplicative perturbation theorems for local $C$-cosine functions in which the operator $C$ may not commute with the bounded perturbation operator $B$ on $X$, which satisfies an estimation that is similar to the condition (2.6) below. In this case, $C^{-1} A(I+B) C$ generates a local $C$-cosine function on $X$ when $C A(I+B) \subset A(I+B) C$ (see [18]). Along this line, Li and Liu also establish some left-multiplicative perturbation theorems for local $C$-cosine functions on $X$ with densely defined generators. In this case, $(I+B) A$ generates a local $C$-cosine function on $X$ when $C^{-1}(I+B) A C=(I+B) A$ (see [20]). Just as continuous work of this topic, Kuo shows that $A+B$ generates a local $C$ cosine function on $X$ when either $B$ is a bounded linear operator from $[D(A)]$ into $R(C)$ such that $R\left(C^{-1} B\right) \subset D(A)$ (see [14]) or $B$ is a bounded linear operator on $X$ which commutes with $C(\cdot)$ on $X$ (see [15] or Theorem 2.13 below). The purpose of this paper is to establish some left and right multiplicative perturbation theorems for local $C$-cosine functions just as results in $[18,20]$ when the generator $A$ of a perturbed local $C$-cosine function $C(\cdot)$ may not be densely defined, the perturbation operator $B$ is only a bounded linear operator from $\overline{D(A)}$ into $R(C)$, and the assumption of $C^{-1}(I+B) A C=(I+B) A$ is not necessary, which together with Theorem 2.13 can be applied to obtain some new Miyadera type additive perturbation theorems just as results in [15] for local $C$-cosine functions (see Theorems 2.14 and 2.16 below). An illustrative example concerning these results is also presented in the final part of this paper.

\section{Perturbation theorems}

In this section, we first note some basic properties of a local $C$-cosine function and known results about connections between the generator of a local $C$-cosine function and strong solutions of the following abstract Cauchy problem:

$$
\operatorname{ACP}(A, f, x, y)\left\{\begin{array}{l}
u^{\prime \prime}(t)=A u(t)+f(t) \quad \text { for } t \in\left(0, T_{0}\right) \\
u(0)=x, u^{\prime}(0)=y
\end{array}\right.
$$

where $x, y \in X$ and $f$ is an $X$-valued function defined on a subset of $\left[0, T_{0}\right)$.

Proposition 2.1. (see [4], [11], [13], [22]). Let $A$ be the generator of a local C-cosine function $C(\cdot)$ on $X$. Then

(2.1) $A$ is closed and $C^{-1} A C=A$;

(2.2) $C(t) x \in D(A)$ and $C(t) A x=A C(t) x$ for all $x \in D(A)$ and $0 \leq t<T_{0}$; 


$$
\int_{0}^{t} \int_{0}^{s} C(r) x d r d s \in D(A) \text { and } A \int_{0}^{t} \int_{0}^{s} C(r) x d r d s=C(t) x-C x \text { for all }
$$
$x \in D(A)$ and $0 \leq t<T_{0}$;

$D(A)=\left\{x \in X \mid C(t) x-C x=\int_{0}^{t} \int_{0}^{s} C(r) y_{x} d r d s\right.$ for all $0 \leq t<T_{0}$ and for some $\left.y_{x} \in X\right\}$ and $A x=y_{x}$ for each $x \in D(A)$;

(2.5) $R(C(t)) \subset \overline{D(A)}$ for $0 \leq t<T_{0}$.

Definition 2.2. Let $A: D(A) \subset X \rightarrow X$ be a closed linear operator in a Banach space $X$ with domain $D(A)$ and range $R(A)$. A function $u:\left[0, T_{0}\right) \rightarrow X$ is called a (strong) solution of $\operatorname{ACP}(A, f, x, y)$, if $u \in C^{2}\left(\left(0, T_{0}\right), X\right) \cap C^{1}\left(\left[0, T_{0}\right), X\right) \cap C\left(\left(0, T_{0}\right),[D(A)]\right)$ and satisfies $\operatorname{ACP}(A, f, x, y)$. Here $[D(A)]$ denotes the Banach space $D(A)$ with norm $|\cdot|$ defined by $|x|=\|x\|+\|A x\|$ for $x \in D(A)$.

Theorem 2.3. (see [11], [13]) A generates a local $C$-cosine function $C(\cdot)$ on $X$ if and only if $C^{-1} A C=A$ and for each $x \in X, A C P(A, C x, 0,0)$ has a unique (strong) solution $u(\cdot, x)$ in $C^{2}\left(\left[0, T_{0}\right), X\right)$. In this case, we have

$$
u(t, x)=j_{1} * C(t) x\left(=\int_{0}^{t} j_{1}(t-s) C(s) x d s\right)
$$

for all $x \in X$ and $0 \leq t<T_{0}$. Here $j_{k}(t)=t^{k} / k$ ! for all $t \in \mathbb{R}$ and $k \in \mathbb{N} \cup\{0\}$.

Proposition 2.4. (see [11], [13]) Let $A$ be the generator of a local $C$-cosine function $C(\cdot)$ on $X, x, y \in X$ and $f \in L_{l o c}^{1}\left(\left[0, T_{0}\right), X\right) \cap C\left(\left(0, T_{0}\right), X\right)$. Then $A C P(A, C f, C x, C y)$ has a (strong) solution $u$ in $C^{2}\left(\left[0, T_{0}\right), X\right)$ if and only if

$$
v(\cdot)=C(\cdot) x+S(\cdot) y+S * f(\cdot) \in C^{2}\left(\left[0, T_{0}\right), X\right) .
$$

In this case, $u=v$ on $\left[0, T_{0}\right)$. Here $S(\cdot)=j_{0} * C(\cdot)$ and $S * f(\cdot)=\int_{0} S(\cdot-s) f(s) d s$.

We next establish a new right-multiplicative perturbation theorem for locally Lipschitz continuous and exponentially Lipschitz continuous local $C$-cosine functions in which $B$ is only a bounded linear operator from $\overline{D(A)}$ into $R(C)$.

Theorem 2.5. Let $C(\cdot)$ be a locally Lipschitz continuous local $C$-cosine function on $X$ with generator $A$. Assume that $B$ is a bounded linear operator from $\overline{D(A)}$ into $R(C)$ such that $C B=B C$ on $\overline{D(A)}$, and for each $0<t_{0}<T_{0}$ there exists an $M_{t_{0}}>0$ such that $\left(S * C^{-1} B f\right)(t) \in D(A)$ and

$$
\left\|A\left(S * C^{-1} B\right)[f(t)-f(s)]\right\| \leq M_{t_{0}} \int_{s}^{t}\|f(r)\| d r
$$

for all $f \in C\left(\left[0, t_{0}\right], \overline{D(A)}\right)$ and $0 \leq s<t \leq t_{0}$. Then $A\left(I+C^{-1} B C\right)$ generates a locally Lipschitz continuous local $C$-cosine function $T(\cdot)$ on $X$ satisfying

$$
T(\cdot) x=C(\cdot) x+A\left(S * C^{-1} B T\right)(\cdot) x \quad \text { on }\left[0, T_{0}\right)
$$

for all $x \in X$. 
Proof. Let $x \in X$ and $0<t_{0}<T_{0}$ be fixed.

We define $U: C\left(\left[0, t_{0}\right], \overline{D(A)}\right) \rightarrow C\left(\left[0, t_{0}\right], \overline{D(A)}\right)$ by

$$
U(f)(\cdot)=C(\cdot) x+A\left(S * C^{-1} B f\right)(\cdot)
$$

on $\left[0, t_{0}\right]$ for all $f \in C\left(\left[0, t_{0}\right], \overline{D(A)}\right)$. Then $U$ is well-defined. By induction, we obtain from (2.6) that

$$
\begin{aligned}
\left\|U^{n} f(t)-U^{n} g(t)\right\| & =\left\|U\left(U^{n-1} f\right)(t)-U\left(U^{n-1} g\right)(t)\right\| \\
& =\left\|A S * C^{-1} B\left(U^{n-1} f-U^{n-1} g\right)(t)\right\| \\
& \leq M_{t_{0}}^{n} \int_{0}^{t} j_{n-1}(t-s)\|f(s)-g(s)\| d s \\
& \leq M_{t_{0}}^{n} j_{n}\left(t_{0}\right)\|f-g\|
\end{aligned}
$$

for all $f, g \in C\left(\left[0, t_{0}\right], \overline{D(A)}\right), 0 \leq t \leq t_{0}$ and $n \in \mathbb{N}$. Here

$$
\|f-g\|=\max _{0 \leq s \leq t_{0}}\|f(s)-g(s)\| .
$$

It follows from the contraction mapping theorem that there exists a unique function $w_{x, t_{0}}$ in $C\left(\left[0, t_{0}\right], \overline{D(A)}\right)$ such that

$$
w_{x, t_{0}}(\cdot)=C(\cdot) x+A S * C^{-1} B w_{x, t_{0}}(\cdot)
$$

on $\left[0, t_{0}\right]$. In this case, we set $w_{x}(t)=w_{x, t_{0}}(t)$ for all $0 \leq t \leq t_{0}<T_{0}$, then $w_{x}(\cdot)$ is a unique function in $C\left(\left[0, T_{0}\right), \overline{D(A)}\right)$ such that

$$
w_{x}(\cdot)=C(\cdot) x+A S * C^{-1} B w_{x}(\cdot)
$$

on $\left[0, T_{0}\right)$. Since

$$
\begin{aligned}
j_{1} * w_{x}(\cdot) & =j_{1} * C(\cdot) x+A j_{1} * S * C^{-1} B w_{x}(\cdot) \\
& =j_{0} * S(\cdot) x+S * C^{-1} B w_{x}(\cdot)-B j_{1} * w_{x}(\cdot)
\end{aligned}
$$

on $\left[0, T_{0}\right)$, we have

$$
(I+B) j_{1} * w_{x}(t)=j_{0} * S(t) x+S * C^{-1} B w_{x}(t) \in D(A)
$$

for all $0 \leq t<T_{0}$. Clearly, $j_{1} * w_{x}$ is the unique function $u_{x}$ in $C^{2}\left(\left[0, T_{0}\right), X\right)$ such that

$$
u_{x}(\cdot)=j_{0} * S(\cdot) x+A S * C^{-1} B u_{x}(\cdot)
$$

on $\left[0, T_{0}\right)$. Since $j_{0} * S(\cdot) x+S * C^{-1} B w_{x}(\cdot) \in C^{2}\left(\left[0, T_{0}\right), X\right)$, we obtain from Proposition 2.4 that

$$
j_{0} * S(\cdot) x+S * C^{-1} B w_{x}(\cdot)=(I+B) j_{1} * w_{x}(\cdot)
$$

is the unique solution of $\operatorname{ACP}\left(A, C x+B w_{x}, 0,0\right)$ in $C^{2}\left(\left[0, T_{0}\right), X\right)$. This implies that

$$
A(I+B) j_{1} * w_{x}+C x+B w_{x}=(I+B) w_{x}
$$

on $\left[0, T_{0}\right)$, and so $A(I+B) j_{1} * w_{x}+C x=w_{x}$ on $\left[0, T_{0}\right)$. Hence, $j_{1} * w_{x}$ is a solution of $\operatorname{ACP}(A(I+B), C x, 0,0)$ in $C^{2}\left(\left[0, T_{0}\right), X\right)$. To prove the uniqueness of solutions of 
$\operatorname{ACP}(A(I+B), C x, 0,0)$.

Suppose that $u \in C\left(\left[0, T_{0}\right), X\right)$ and satisfies $A(I+B) j_{1} * u+C x=u$ on $\left[0, T_{0}\right)$. Then

$$
\begin{aligned}
j_{1} *\left(S * u-S * j_{0} C x\right) & =j_{1} * S * A(I+B) j_{1} * u \\
& =A j_{1} * S *(I+B) j_{1} * u \\
& =S *(I+B) j_{1} * u-C j_{1} *(I+B) j_{1} * u \\
& =S * j_{1} * u+S * B j_{1} * u-C j_{1} *(I+B) j_{1} * u
\end{aligned}
$$

on $\left[0, T_{0}\right)$, and so $-S * j_{2}(\cdot) C x=S * B j_{1} * u(\cdot)-C j_{1} *(I+B) j_{1} * u(\cdot)$ on $\left[0, T_{0}\right)$. Hence,

$$
\begin{aligned}
-S * j_{0}(\cdot) x & =\left(S * C^{-1} B j_{1} * u\right)^{\prime \prime}(\cdot)-(I+B) j_{1} * u(\cdot) \\
& =A S * C^{-1} B j_{1} * u(\cdot)+B j_{1} * u(\cdot)-(I+B) j_{1} * u(\cdot) \\
& =A S * C^{-1} B j_{1} * u(\cdot)-j_{1} * u(\cdot)
\end{aligned}
$$

on $\left[0, T_{0}\right)$, which implies that $j_{1} * u(\cdot)=S * j_{0}(\cdot) x+A S * C^{-1} B j_{1} * u(\cdot)$ on $\left[0, T_{0}\right)$. Consequently, $j_{1} * u=j_{1} * w_{x}$ on $\left[0, T_{0}\right)$ or equivalently, $u=w_{x}$ on $\left[0, T_{0}\right)$. Clearly, $A(I+B)$ is closed and $A(I+B) C=C A(I+B)$ on $D(A(I+B))$. It follows from Proposition 2.4 that $C^{-1} A(I+B) C$ generates a local $C$-cosine function $T(\cdot)$ on $X$ satisfying (2.7) for all $x \in X$. Just as in the proof of [27, Theorem 2.5], we have $C^{-1} A(I+B) C=A\left(I+C^{-1} B C\right)$. By (2.6), $T(\cdot)$ is also locally Lipschitz continuous.

Since the condition (2.6) in the proof of Theorem 2.5 is only used to show that $T(\cdot)$ is locally Lipschitz continuous. By slightly modifying the proof of Theorem 2.5, we can obtain the next right-multiplicative perturbation theorem for local $C$-cosine functions without the local Lipschitz continuity.

Theorem 2.6. Let $C(\cdot)$ be a local $C$-cosine function on $X$ with generator $A$. Assume that $B$ is a bounded linear operator from $\overline{D(A)}$ into $R(C)$ such that $C B=B C$ on $\overline{D(A)}$, and for each $0<t_{0}<T_{0}$ there exists an $M_{t_{0}}>0$ such that $\left(S * C^{-1} B f\right)(t) \in$ $D(A)$ and

$$
\left\|A\left(S * C^{-1} B f\right)(t)\right\| \leq M_{t_{0}} \int_{0}^{t}\|f(s)\| d s
$$

for all $f \in C\left(\left[0, t_{0}\right], \overline{D(A)}\right)$ and $0 \leq t \leq t_{0}$. Then $A\left(I+C^{-1} B C\right)$ generates a local $C$-cosine function $T(\cdot)$ on $X$ satisfying (2.7)

Corollary 2.7. Let $C(\cdot)$ be a locally Lipschitz continuous local $C$-cosine function on $X$ with generator $A$. Assume that $B$ is a bounded linear operator from $\overline{D(A)}$ into $R(C)$ such that $C B=B C$ on $\overline{D(A)}$ and $C^{-1} B x \in \overline{D(A)}$ for all $x \in \overline{D(A)}$. Then $A\left(I+C^{-1} B C\right)$ generates a locally Lipschitz continuous local $C$-cosine function $T(\cdot)$ on $X$ satisfying (2.7) for all $x \in X$. Moreover, $T(\cdot)$ is exponentially Lipschitz continuous if $C(\cdot)$ is.

Proof. Clearly, it suffices to show that for each $0<t_{0}<T_{0}$ there exists an $M_{t_{0}}>0$ such that $(2.6)$ holds for all $f \in C\left(\left[0, t_{0}\right], \overline{D(A)}\right)$ and $0 \leq s<t \leq t_{0}$. Suppose that 
$C_{1}(t)$ denotes the restriction of $C(t)$ to $\overline{D(A)}, C_{1}^{\prime}(t)$ the strong derivative of $C_{1}(t)$ on $\overline{D(A)}$ for all $0 \leq t<T_{0}$, and $D^{2}$ the second order derivative of a function. Then $C_{1}(t) x=C x+A j_{0} * S(t) x$ and $C_{1}^{\prime}(t) x=A S(t) x$ for all $x \in \overline{D(A)}$ and $0 \leq t<T_{0}$. In particular, $A S(\cdot)$ is a strongly continuous family of bounded linear operators on $\overline{D(A)}$, which is also exponentially bounded if $C(\cdot)$ is exponentially Lipschitz continuous. Let $0<t_{0}<T_{0}$ be given, then $S * C^{-1} B f(\cdot)$ is twice continuously differentiable on $\left[0, t_{0}\right]$,

$$
D^{2}\left(S * C^{-1} B f\right)(\cdot)=A S * C^{-1} B f(\cdot)+B f(\cdot)=C_{1}^{\prime} * C^{-1} B f(\cdot)+B f(\cdot)
$$

on $\left[0, t_{0}\right]$ and

$$
\begin{aligned}
\left\|A\left(S * C^{-1} B[f(t)-f(s)]\right)\right\| & =\left\|C_{1}^{\prime} * C^{-1} B[f(t)-f(s)]\right\| \\
& \leq \sup _{0 \leq r \leq t_{0}}\|A S(r)\|\left\|C^{-1} B\right\| \int_{s}^{t}\|f(r)\| d r
\end{aligned}
$$

for all $f \in C\left(\left[0, t_{0}\right], \overline{D(A)}\right)$ and $0 \leq s<t \leq t_{0}$. It follows from Theorem 2.3 that $A\left(I+C^{-1} B C\right)$ generates a locally Lipschitz continuous local $C$-cosine function $T(\cdot)$ on $X$ satisfying (2.7) for all $x \in X$. Combining the local Lipschitz continuity of $C^{-1} B T(\cdot)$ with the exponential boundedness of $A S(\cdot)$, we get that $\left.A S * C^{-1} B T\right)(\cdot)$ is exponentially Lipschitz continuous if $C(\cdot)$ is. Consequently, $T(\cdot)$ is exponentially Lipschitz continuous if $C(\cdot)$ is.

Corollary 2.8. Let $C(\cdot)$ be a local $C$-cosine function on $X$ with generator $A$. Assume that $B$ is a bounded linear operator from $\overline{D(A)}$ into $R(C)$ such that $C B=B C$ on $\overline{D(A)}$ and $C^{-1} B x \in D(A)$ for all $x \in \overline{D(A)}$. Then $A\left(I+C^{-1} B C\right)$ generates a local $C$-cosine function $T(\cdot)$ on $X$ satisfying

$$
T(\cdot) x=C(\cdot) x+S * A C^{-1} B T(\cdot) x \quad \text { on }\left[0, T_{0}\right)
$$

for all $x \in X$. Moreover, $T(\cdot)$ is also exponentially bounded (resp., norm continuous) if $C(\cdot)$ is.

Proof. By the assumption of $C^{-1} B x \in D(A)$ for all $x \in \overline{D(A)}$, we can apply the following estimation to replace the condition (2.8):

$$
\left\|\left(S * A C^{-1} B f(t)\right)\right\| \leq \sup _{0 \leq r \leq t_{0}}\|S(r)\|\left\|A C^{-1} B\right\| \int_{0}^{t}\|f(r)\| d r
$$

for all $f \in C\left(\left[0, t_{0}\right], \overline{D(A)}\right)$ and $0 \leq t \leq t_{0}$. Clearly, $S(\cdot) A C^{-1} B$ is also exponentially bounded (resp., norm continuous) if $C(\cdot)$ is. By $(2.9)$ and the boundedness of $A C^{-1} B$, we have

$$
T(\cdot) x=C(\cdot) x+S A C^{-1} B * T(\cdot) x \quad \text { on }\left[0, T_{0}\right)
$$

for all $x \in X$, which together with Gronwall's inequality implies that $T(\cdot)$ is exponentially bounded (resp., norm continuous) if $C(\cdot)$ is.

When $\rho\left(\left(I+C^{-1} B C\right) A\right)$ (resolvent set of $\left.\left(I+C^{-1} B C\right) A\right)$ is nonempty, we can apply Theorem 2.5 to obtain the next left-multiplicative perturbation theorem concerning locally Lipschitz continuous local $C$-cosine functions on $X$ in which the generator $A$ of a perturbed local $C$-cosine function may not be densely defined, $B$ is 
only a bounded linear operator from $\overline{D(A)}$ into $R(C)$, and $C^{-1}(I+B) A C$ and $(I+B) A$ both may not be equal.

Theorem 2.9. Under the assumptions of Theorem 2.5. Assume that $\rho\left(\left(I+C^{-1} B C\right) A\right)$ is nonempty. Then $\left(I+C^{-1} B C\right) A$ generates a locally Lipschitz continuous local $C$ cosine function $U(\cdot)$ on $X$ satisfying

$$
\begin{aligned}
& U(\cdot) x \\
= & C x+\left[\lambda-\left(I+C^{-1} B C\right) A\right]\left(I+C^{-1} B C\right) j_{1} * T(\cdot) A\left[\lambda-\left(I+C^{-1} B C\right) A\right]^{-1} x
\end{aligned}
$$

on $\left[0, T_{0}\right)$ for all $x \in X$. Here $\lambda \in \rho\left(\left(I+C^{-1} B C\right) A\right)$ is fixed and $T(\cdot)$ is given as in (2.7).

Proof. Just as in the proof of [27, Theorem 2.9], we have

$$
\left(I+C^{-1} B C\right) A C x=C\left(I+C^{-1} B C\right) A x
$$

for all $x \in D\left(\left(I+C^{-1} B C\right) A\right)$. We set $P=I+C^{-1} B C$ and

$$
u_{x}(\cdot)=C x+(\lambda-P A) P j_{1} * T(\cdot) A(\lambda-P A)^{-1} x
$$

on $\left[0, T_{0}\right)$ for all $x \in X$, then $u_{x} \in C\left(\left[0, T_{0}\right), X\right)$ and

$$
\begin{aligned}
& A(\lambda-P A)^{-1} u_{x}(\cdot) \\
= & A(\lambda-P A)^{-1} C x+A\left(P j_{1} * T(\cdot)\right) A(\lambda-P A)^{-1} x \\
= & A(\lambda-P A)^{-1} C x+T(\cdot) A(\lambda-P A)^{-1} x-C A(\lambda-P A)^{-1} x \\
= & A(\lambda-P A)^{-1} C x+T(\cdot) A(\lambda-P A)^{-1} x-A(\lambda-P A)^{-1} C x \\
= & T(\cdot) A(\lambda-P A)^{-1} x
\end{aligned}
$$

on $\left[0, T_{0}\right)$, and so

$$
P A(\lambda-P A)^{-1} j_{1} * u_{x}(\cdot)=P j_{1} * T(\cdot) A(\lambda-P A)^{-1} x
$$

on $\left[0, T_{0}\right)$. Hence,

$$
\begin{aligned}
-j_{1} * u_{x}(\cdot)+\lambda(\lambda-P A)^{-1} j_{1} * u_{x}(\cdot) & =P A(\lambda-P A)^{-1} j_{1} * u_{x}(\cdot) \\
& =P j_{1} * T(\cdot) A(\lambda-P A)^{-1} x \\
& =(\lambda-P A)^{-1} u_{x}(\cdot)-(\lambda-P A)^{-1} C x
\end{aligned}
$$

on $\left[0, T_{0}\right)$, which implies that $j_{1} * u_{x}(t) \in D(P A)$ for all $0 \leq t<T_{0}$. Consequently,

$$
P A(\lambda-P A)^{-1} j_{1} * u_{x}(t) \in D(P A)
$$

for all $0 \leq t<T_{0}$ and $P A j_{1} * u_{x}=u_{x}-C x$ on $\left[0, T_{0}\right)$. This shows that $j_{1} * u_{x}$ is a solution of $\operatorname{ACP}(P A, C x, 0,0)$ in $C^{2}\left(\left[0, T_{0}\right), X\right)$. In order to show the uniqueness. Suppose that $v \in C\left(\left[0, T_{0}\right), X\right)$ and $v=P A j_{1} * v$ on $\left[0, T_{0}\right)$. We set $u=A(\lambda-P A)^{-1} v$ on $\left[0, T_{0}\right)$, then

$$
\begin{aligned}
P j_{1} * u & =P A(\lambda-P A)^{-1} j_{1} * v \\
& =(\lambda-P A)^{-1} P A j_{1} * v \\
& =(\lambda-P A)^{-1} v
\end{aligned}
$$


on $\left[0, T_{0}\right)$, and so $A P j_{1} * u=A(\lambda-P A)^{-1} v=u$ on $\left[0, T_{0}\right)$. Hence, $u=0$ on $\left[0, T_{0}\right)$, which implies that $(\lambda-P A)^{-1} v=0$ on $\left[0, T_{0}\right)$ or equivalently, $v=0$ on $\left[0, T_{0}\right)$. We conclude from Theorem 2.3 that $\left(I+C^{-1} B C\right) A$ generates a local $C$-cosine function $U(\cdot)$ on $X$ satisfying (2.11) for all $x \in X$. Clearly, for each $y \in X$,

$$
(P A) P j_{1} * T(\cdot) y=P(A P) j_{1} * T(\cdot) y=P T(\cdot) y-P C y
$$

on $\left[0, T_{0}\right)$. It follows from the right-hand side of $(2.11)$ that $U(\cdot)$ is also locally Lipschitz continuous.

By slightly modifying the proof of Theorem 2.9, we can obtain the next leftmultiplicative perturbation theorem for local $C$-cosine functions in which the generator $A$ of a perturbed local $C$-cosine function may not be densely defined, $B$ is only a bounded linear operator from $\overline{D(A)}$ into $R(C)$, and $C^{-1}(I+B) A C$ and $(I+B) A$ both may not be equal.

Theorem 2.10. Under the assumptions of Theorem 2.6. Assume that $\rho\left(\left(I+C^{-1} B C\right) A\right)$ is nonempty. Then $\left(I+C^{-1} B C\right) A$ generates a local $C$-cosine function $U(\cdot)$ on $X$ satisfying (2.11) for all $x \in X$. Moreover, $U(\cdot)$ is exponentially bounded (resp., norm continuous, locally Lipschitz continuous, or exponentially Lipschitz continuous) if $T(\cdot)$ is. Here $T(\cdot)$ is given as in (2.7).

Corollary 2.11. Under the assumptions of Corollary 2.7.

Assume that $\rho\left(\left(I+C^{-1} B C\right) A\right)$ is nonempty. Then $\left(I+C^{-1} B C\right) A$ generates a locally Lipschitz continuous local $C$-cosine function $U(\cdot)$ on $X$ satisfying (2.11) for all $x \in X$. Moreover, $U(\cdot)$ is exponentially Lipschitz continuous if $C(\cdot)$ is.

Corollary 2.12. Under the assumptions of Corollary 2.8.

Assume that $\rho\left(\left(I+C^{-1} B C\right) A\right)$ is nonempty. Then $\left(I+C^{-1} B C\right) A$ generates a local $C$-cosine function $U(\cdot)$ on $X$ satisfying (2.11) for all $x \in X$. Moreover, $U(\cdot)$ is also exponentially bounded (resp., norm continuous) if $C(\cdot)$ is.

Theorem 2.13. (see [15]) Let $A$ be the generator of a local $C$-cosine function $C(\cdot)$ on $X$. Assume that $B$ is a bounded linear operator on $X$ which commutes with $C(\cdot)$ on $X$. Then $A+B$ is the generator of a local $C$-cosine function $T_{B}(\cdot)$ on $X$ satisfying

$$
T_{B}(t) x=\sum_{n=0}^{\infty} \int_{0}^{t} j_{n-1}(s) j_{n}(t-s) C(|t-2 s|) B^{n} x d s
$$

for all $x \in X$ and $0 \leq t<T_{0}$.

Combining Theorem 2.10 with Theorem 2.13, the next new result concerning the additive perturbations of a local $C$-cosine function on $X$ is also attained in which the generator of a perturbed local $C$-cosine function may not be densely defined.

Theorem 2.14. Let $C(\cdot)$ be a local $C$-cosine function on $X$ with generator $A$, and let $B$ be a bounded linear operator from $[D(A)]$ into $R\left(C^{2}\right)$ such that $C B=B C$ on $D(A)$. Assume that $\rho_{C}(A)$ and $\rho(A+B)$ both are nonempty, and for each $0<t_{0}<T_{0}$ there exists an $M_{t_{0}}>0$ such that

$$
\left|S * C^{-2} B f(t)\right| \leq M_{t_{0}} \int_{0}^{t}|f(s)| d s
$$


for all $f \in C\left(\left[0, t_{0}\right],[D(A)]\right)$ and $0 \leq t \leq t_{0}$. Then $A+B$ generates a local $C$-cosine function $V(\cdot)$ on $X$.

Proof. Let $\lambda \in \rho_{C}(A)$ be fixed. We set $\widetilde{B}=C^{-1} B(A-\lambda)^{-1} C$ and $C(-t)=C(t)$ for all $0 \leq t<T_{0}$. Then $\widetilde{B}$ is a bounded linear operator from $X$ into $R(C)$ such that $C \widetilde{B}=\widetilde{B} C, A-\lambda$ is the generator of the local $C$-cosine function $T_{-\lambda}(\cdot)$ on $X$ satisfying

$$
j_{0} * T_{-\lambda}(t) x=\sum_{n=0}^{\infty} \int_{0}^{t} j_{n-1}(s) j_{n}(t-s) S(t-2 s)(-\lambda)^{n} x d s
$$

for all $x \in X$ and $0 \leq t<T_{0}$, and $(A-\lambda)^{-1} C^{2}=C(A-\lambda)^{-1} C$. Here

$$
\int_{0}^{t} j_{-1}(s) j_{0}(t-s) S(t-2 s) x d s=S(t) x .
$$

Since the norm $|\cdot|_{A-\lambda}$ on $D(A)$ defined by $|x|_{A-\lambda}=\|x\|+\|(A-\lambda) x\|$ for all $x \in D(A)$, is equivalent to $|\cdot|$, we may assume that $(2.12)$ holds under $|\cdot|_{A-\lambda}$. Since

$$
\left(I+C^{-1} \widetilde{B} C\right)(A-\lambda)=A-\lambda+B
$$

and $\rho(A+B)$ is nonempty we have $\rho\left(\left(I+C^{-1} \widetilde{B} C\right)(A-\lambda)\right)$ is also nonempty. It is not difficult to see that

$$
\begin{aligned}
& \int_{0}^{t} j_{n-1}(s) j_{n}(t-s) S(t-2 s) x d s \\
= & \sum_{k=0}^{n} \frac{(n-1+k) !}{(n-1) ! k !}(-1)^{k} \frac{1}{2^{n+k}}\left[j_{n-k}\left(j_{n-1+k} * S\right)\right](t) x \\
+ & \sum_{k=0}^{n-1} \frac{(n+k) !}{n ! k !}(-1)^{k} \frac{1}{2^{n+k+1}}\left[j_{n-1-k}\left(j_{n+k} * S\right)\right](t) x
\end{aligned}
$$

for each $n \in \mathbb{N}, x \in X$ and $0 \leq t<T_{0}$. Let $0<t_{0}<T_{0}$ and $f \in C\left(\left[0, t_{0}\right], X\right)$ be fixed. Then

$$
\begin{aligned}
& {\left[j_{n-k}\left(j_{n-1+k} * S\right)\right] * C^{-1} \widetilde{B} f(t) } \\
= & \int_{0}^{t} j_{n-k}(t-s)\left(j_{n-1+k} * S\right)(t-s) C^{-1} \widetilde{B} f(s) d s \\
= & \frac{1}{(n-k) !} \sum_{m=0}^{n-k}\left(\begin{array}{c}
n-k \\
m
\end{array}\right)(-1)^{m} t^{n-k-m} \int_{0}^{t} j_{n-1+k} * S(t-s) C^{-1} \widetilde{B} s^{m} f(s) d s \\
= & \left.\sum_{m=0}^{n-k}(-1)^{m} j_{n-k-m}(t) j_{n-1+k} * S * C^{-1} \widetilde{B} j_{m} f\right)(t) \\
= & \sum_{m=0}^{n-k}(-1)^{m} j_{n-k-m}(t) S * C^{-1} \widetilde{B}\left[j_{n-1+k} *\left(j_{m} f\right)\right](t)
\end{aligned}
$$


and

$$
\begin{aligned}
& {\left[j_{n-1-k}\left(j_{n+k} * S\right)\right] * C^{-1} \widetilde{B} f(t) } \\
= & \int_{0}^{t} j_{n-1-k}(t-s)\left(j_{n+k} * S\right)(t-s) C^{-1} \widetilde{B} f(s) d s \\
= & \frac{1}{(n-1-k) !} \sum_{m=0}^{n-1-k}\left(\begin{array}{c}
n-1-k \\
m
\end{array}\right)(-1)^{m} t^{n-1-k-m} \int_{0}^{t} j_{n+k} * S(t-s) C^{-1} \widetilde{B} s^{m} f(s) d s \\
= & \sum_{m=0}^{n-1-k}(-1)^{m} j_{n-1-k-m}(t) j_{n+k} * S *\left(C^{-1} \widetilde{B} j_{m} f\right)(t) \\
= & \sum_{m=0}^{n-1-k}(-1)^{m} j_{n-1-k-m}(t) S * C^{-1} \widetilde{B}\left[j_{n+k} *\left(j_{m} f\right)\right](t)
\end{aligned}
$$

for all $0 \leq t \leq t_{0}$. By (2.12), we have

$$
\begin{aligned}
& \left\|(A-\lambda) j_{n-k-m}(t) S * C^{-1} \widetilde{B}\left[j_{n-1+k} *\left(j_{m} f\right)\right](t)\right\| \\
\leq & j_{n-k-m}\left(t_{0}\right)\left\|(A-\lambda) S * C^{-1} \widetilde{B}\left[j_{n-1+k} *\left(j_{m} f\right)\right](t)\right\| \\
= & j_{n-k-m}\left(t_{0}\right)\left\|(A-\lambda) S * C^{-2} B(A-\lambda)^{-1} C\left[j_{n-1+k} *\left(j_{m} f\right)\right](t)\right\| \\
\leq & j_{n-k-m}\left(t_{0}\right) M_{t_{0}} \int_{0}^{t}\left|(A-\lambda)^{-1} C\left[j_{n-1+k} *\left(j_{m} f\right)\right](s)\right|_{A-\lambda} d s \\
\leq & j_{n-k-m}\left(t_{0}\right) M_{t_{0}}\left(\left\|(A-\lambda)^{-1} C\right\|+\|C\|\right) \int_{0}^{t}\left\|\left[j_{n-1+k} *\left(j_{m} f\right)\right](s)\right\| d s
\end{aligned}
$$

for all $0 \leq t \leq t_{0}$. Since

$$
\begin{aligned}
& \int_{0}^{t}\left\|\left[j_{n-1+k} *\left(j_{m} f\right)\right](s)\right\| d s \\
\leq & \int_{0}^{t} j_{n-1+k}(s) j_{m}(s) \int_{0}^{s}\|f(s)\| d s \\
= & \frac{(n+k-1+m) !}{(n-1+k) ! m !}\left[j_{n+k+m}(t) \int_{0}^{t}\|f(r)\| d r-\int_{0}^{t}\|f(s)\| d s\right] \\
\leq & \frac{(n+k-1+m) !}{(n-1+k) ! m !} j_{n+k+m}(t) \int_{0}^{t}\|f(r)\| d r
\end{aligned}
$$

for all $0 \leq t \leq t_{0}$, we have

$$
\begin{aligned}
& \|(A-\lambda) j_{n-k-m}(t) S *\left(C^{-1} \widetilde{B}\left[j_{n-1+k} *\left(j_{m} f\right)\right](t) \|\right. \\
\leq & j_{n-k-m}\left(t_{0}\right) M_{t_{0}}\left(\left\|(A-\lambda)^{-1} C\right\|+\|C\|\right) \frac{(n+k-1+m) !}{(n-1+k) ! m !} j_{n+k+m}(t) \int_{0}^{t}\|f(r)\| d r
\end{aligned}
$$


for all $0 \leq t \leq t_{0}$. Similarly, we can apply (2.12) and (2.15) to obtain

$$
\begin{aligned}
& \|(A-\lambda) j_{n-1-k-m}(t) S *\left(C^{-1} \widetilde{B}\left[j_{n+k} *\left(j_{m} f\right)\right](t) \|\right. \\
\leq & j_{n-1-k-m}\left(t_{0}\right) M_{t_{0}}\left(\left\|(A-\lambda)^{-1} C\right\|+\|C\|\right) \int_{0}^{t}\left\|\left[j_{n+k} *\left(j_{m} f\right)\right](s)\right\| d s \\
\leq & j_{n-1-k-m}\left(t_{0}\right) M_{t_{0}}\left(\left\|(A-\lambda)^{-1} C\right\|+\|C\|\right) \frac{(n+k+m) !}{(n+k) ! m !} j_{n+k+m-1}(t) \int_{0}^{t}\|f(r)\| d r
\end{aligned}
$$

for all $0 \leq t \leq t_{0}$. By $(2.13)$, we have

$$
\begin{aligned}
& j_{0} * T_{-\lambda} * C^{-1} \widetilde{B} f(t)=S * C^{-1} \widetilde{B} f(t)+ \\
& \sum_{n=1}^{\infty}(-\lambda)^{n} \sum_{k=0}^{n} \frac{(n-1+k) !}{(n-1) ! k !}(-1)^{k} \frac{1}{2^{n+k}}\left[j_{n-k}\left(j_{n-1+k} * S\right)\right] * C^{-1} \widetilde{B} f(t) \\
& +\sum_{n=1}^{\infty}(-\lambda)^{n} \sum_{k=0}^{n-1} \frac{(n+k) !}{n ! k !}(-1)^{k} \frac{1}{2^{n+k+1}}\left[j_{n-1-k}\left(j_{n+k} * S\right)\right] * C^{-1} \widetilde{B} f(t)
\end{aligned}
$$

for all $0 \leq t \leq t_{0}$. By (2.14) and (2.18), we have

$$
\begin{aligned}
& \left\|(A-\lambda) \sum_{k=0}^{n} \frac{(n-1+k) !}{(n-1) ! k !}(-1)^{k} \frac{1}{2^{n+k}}\left[j_{n-k}\left(j_{n-1+k} * S\right)\right] * C^{-1} \widetilde{B} f(t)\right\| \\
= & \|(A-\lambda) \sum_{k=0}^{n} \sum_{m=0}^{n-k} \frac{(n-1+k) !}{(n-1) ! k !}(-1)^{k+m} \frac{1}{2^{n+k}} j_{n-k-m}(t) S \\
& * C^{-1} \widetilde{B}\left[j_{n-1+k} *\left(j_{m} f\right)\right](t) \| \\
\leq & \sum_{k=0}^{n} \sum_{m=0}^{n-k} \frac{(n-1+k) !}{(n-1) ! k !} \frac{1}{2^{n+k}} \frac{(n-1+k+m) !}{(n-1+k) ! m !} j_{n-k-m}\left(t_{0}\right) M_{t_{0}}\left(\left\|(A-\lambda)^{-1} C\right\|\right. \\
& +\|C\|) j_{n+k+m}(t) \int_{0}^{t}\|f(r)\| d r \\
\leq & \sum_{k=0}^{n} \frac{t_{0}^{2 n}}{n ! k ! 2^{n+k}} \sum_{m=0}^{n-k} \frac{1}{m !} M_{t_{0}}\left(\left\|(A-\lambda)^{-1} C\right\|+\|C\|\right) \int_{0}^{t}\|f(r)\| d r \\
\leq & \frac{t_{0}^{2 n}}{n ! 2^{n}} e^{1 / 2} e M_{t_{0}}\left(\left\|(A-\lambda)^{-1} C\right\|+\|C\|\right) \int_{0}^{t}\|f(r)\| d r .
\end{aligned}
$$


Similarly, we can apply (2.15) and (2.19) to show that

$$
\begin{aligned}
& \left\|(A-\lambda) \sum_{k=0}^{n-1} \frac{(n-1+k) !}{n ! k !}(-1)^{k} \frac{1}{2^{n+k+1}}\left[j_{n-1-k}\left(j_{n+k} * S\right)\right] * C^{-1} \widetilde{B} f(t)\right\| \\
= & \|(A-\lambda) \sum_{k=0}^{n-1} \sum_{m=0}^{n-1-k} \frac{(n+k) !}{n ! k !}(-1)^{k} \frac{1}{2^{n+k+1}}(-1)^{m} j_{n-1-k-m}(t) S \\
& * C^{-1} \widetilde{B}\left[j_{n+k} *\left(j_{m} f\right)\right](t) \| \\
\leq & \sum_{k=0}^{n-1} \sum_{m=0}^{n-1-k} \frac{(n+k) !}{n ! k !} \frac{1}{2^{n+k+1}} \frac{(n+k+m) !}{(n+k) ! m !} j_{n-1-k-m}\left(t_{0}\right) M_{t_{0}}\left(\left\|(A-\lambda)^{-1} C\right\|\right. \\
& +\|C\|) j_{n+k+m-1}(t) \int_{0}^{t}\|f(r)\| d r \\
\leq & \sum_{k=0}^{n-1} \frac{t_{0}^{2 n}}{(n-1) ! k ! 2^{n+k}} \sum_{m=0}^{n-1-k} \frac{1}{m !} M_{t_{0}}\left(\left\|(A-\lambda)^{-1} C\right\|+\|C\|\right) \int_{0}^{t}\|f(r)\| d r \\
\leq & \frac{t_{0}^{2 n}}{(n-1) ! 2^{n}} e^{1 / 2} e M_{t_{0}}\left(\left\|(A-\lambda)^{-1} C\right\|+\|C\|\right) \int_{0}^{t}\|f(r)\| d r .
\end{aligned}
$$

Combining (2.20)-(2.22), we get that there exists an $\widetilde{M_{t_{0}}}>0$ such that

$$
\left\|(A-\lambda) j_{0} * T_{-\lambda} * C^{-1} \widetilde{B} f(t)\right\| \leq \widetilde{M_{t_{0}}} \int_{0}^{t}\|f(s)\| d s
$$

for all $f \in C\left(\left[0, t_{0}\right], X\right)$ and $0 \leq t \leq t_{0}$. It follows from Theorem 2.5 that $A+B-\lambda$ generates a local $C$-cosine function $U(\cdot)$ on $X$, which implies that $A+B$ generates a local $C$-cosine function $V(\cdot)$ on $X$.

Just as in the proof of Corollary 2.8, we can apply Theorems 2.13 and 2.14 to obtain the next corollary.

Corollary 2.15. Let $C(\cdot)$ be a local $C$-cosine function on $X$ with generator $A$, and let $B$ be a bounded linear operator from $[D(A)]$ into $R\left(C^{2}\right)$ such that $C B=B C$ on $D(A)$ and $C^{-2} B x \in D(A)$ for all $x \in D(A)$. Assume that $\rho_{C}(A)$ and $\rho(A+B)$ both are nonempty. Then $A+B$ generates a local $C$-cosine function $V(\cdot)$ on $X$ given as in the proof of Theorem 2.14. Moreover, $V(\cdot)$ is exponentially bounded (resp., norm continuous) if $C(\cdot)$ is.

By slightly modifying the proof of Theorem 2.14, the following additive perturbation results are also attained when $\widetilde{B}$ denotes the restriction of $B(A-\lambda)^{-1}$ to $\overline{D(A)}$, and the assumptions that $B$ is a bounded linear operator from $[D(A)]$ into $R\left(C^{2}\right)$ and $\rho_{C}(A)$ is nonempty are replaced by assuming that $B$ is a bounded linear operator from $[D(A)]$ into $R(C)$ and $\rho(A)$ is nonempty.

Theorem 2.16. Let $C(\cdot)$ be a local $C$-cosine function on $X$ with generator $A$, and let $B$ be a bounded linear operator from $[D(A)]$ into $R(C)$ such that $C B=B C$ on $D(A)$. 
Assume that $\rho(A)$ and $\rho(A+B)$ both are nonempty, and for each $0<t_{0}<T_{0}$ there exists an $M_{t_{0}}>0$ such that

$$
\left|S * C^{-1} B f(t)\right| \leq M_{t_{0}} \int_{0}^{t}|f(s)| d s
$$

for all $f \in C\left(\left[0, t_{0}\right],[D(A)]\right)$ and $0 \leq t \leq t_{0}$. Then $A+B$ generates a local $C$-cosine function on $X$.

Corollary 2.17. Let $C(\cdot)$ be a local $C$-cosine function on $X$ with generator $A$, and let $B$ be a bounded linear operator from $[D(A)]$ into $R(C)$ such that $C B=B C$ on $D(A)$ and $C^{-1} B x \in D(A)$ for all $x \in D(A)$. Assume that $\rho(A)$ and $\rho(A+B)$ both are nonempty. Then $A+B$ generates a local $C$-cosine function on $X$, which is also exponentially bounded (resp., norm continuous) if $C(\cdot)$ is.

Remark 2.18. The conclusions of Corollaries 2.7 and 2.11 are still true when the assumption that $R\left(C^{-1} B\right) \subset \overline{D(A)}$ is replaced by assuming that

$$
R\left(C^{-1} B\right) \subset\left\{x \in X \mid C(\cdot) x \in C^{1}\left(\left[0, T_{0}\right), X\right)\right\} .
$$

We end this paper with a simple illustrative example.

Example 2.19. Let $X=L^{\infty}(\mathbb{R})$, and $A_{0}: D\left(A_{0}\right) \subset X \rightarrow X$ be defined by

$$
D\left(A_{0}\right)=W^{1, \infty}(\mathbb{R})
$$

and $A_{0} f=-f^{\prime}$ for all $f \in D\left(A_{0}\right)$, then $A=A_{0}^{2}$ generates a locally Lipschitz continuous local $C$-cosine function $C(\cdot)\left(=\left\{C(t) \mid 0 \leq t<T_{0}\right\}\right)$ on $X$ and

$$
\overline{D(A)}=\overline{W^{2, \infty}(\mathbb{R})}=C_{0}(\mathbb{R})
$$

(see [1, Example 3.15.5] and [17, Theorem 18.3]). Here $C=\left(\lambda-A_{0}\right)^{-1}$ with $\lambda \in \rho\left(A_{0}\right)$ and $0<T_{0} \leq \infty$ are fixed. Applying Corollary 2.7, we get that $A\left(I+C^{-1} B C\right)$ generates a locally Lipschitz continuous local $C$-cosine function $T(\cdot)$ on $L^{\infty}(\mathbb{R})$ satisfying (2.7) when $B$ is a bounded linear operator from $C_{0}(\mathbb{R})$ into $W^{1, \infty}(\mathbb{R})$ such that $\left(\lambda-A_{0}\right)^{-1} B=B\left(\lambda-A_{0}\right)^{-1}$ on $C_{0}(\mathbb{R})$ and $R\left(\left(\lambda-A_{0}\right) B\right) \subset C_{0}(\mathbb{R})$.

\section{References}

[1] Arendt, W., Batty, C.J.K., Hieber, H., Neubrander, F., Vector-Valued Laplace Transforms and Cauchy Problems, 96, Birkhäuser Verlag, Basel-Boston-Berlin, 2001.

[2] Engel, K.-J., On singular perturbations of second order Cauchy problems, Pacific J. Math., 152(1992), 79-91.

[3] Fattorini, H.O., Second Order Linear Differential Equations in Banach Spaces, 108, North-Holland Math. Stud., North-Holland, Amsterdam, 1985.

[4] Gao, M.C., Local C-semigroups and C-cosine functions, Acta Math. Sci., 19(1999), 201213.

[5] Goldstein, J.A., Semigroups of Linear Operators and Applications, Oxford Univ. Press, Oxford, 1985.

[6] Hieber, M., Integrated Semigroups and Differential Operators on $L^{p}$, Ph.D. Dissertation, Tubingen, 1989. 
[7] Huang, F., Huang, T., Local C-cosine family theory and application, Chin. Ann. Math., 16(1995), 213-232.

[8] Kellerman, H., Hiebe, M., Integrated semigroups, J. Funct. Anal., 84(1989), 160-180.

[9] Kostic, M., Perturbation theorems for convoluted C-semigroups and cosine functions, Bull. Ci. Sci. Math., 35(2010), 25-47.

[10] Kostic, M., Generalized Semigroups and Cosine Functions, Mathematical Institute SANU, Belgrade, 2011.

[11] Kostic, M., Abstract Volterra Integro-Differential Equations, CRC Press, Boca Raton, FI., 2015.

[12] Kostic, M., Abstract Degenerate Volterra Integro-Differential Equations: Linear Theory and Applications, Book Manuscript, 2016.

[13] Kuo, C.-C., Shaw, S.-Y., C-cosine functions and the abstract Cauchy problem I, II, J. Math. Anal. Appl., 210(1997), 632-646, 647-666.

[14] Kuo, C.-C., On perturbation of local integrated cosine functions, Taiwanese J. Math., 11(2012), 1613-1628.

[15] Kuo, C.-C., Perturbations of local C-cosine functions, Stud. Univ. Babeş-Bolyai Math., 65(2020), 585-597.

[16] Kuo, C.-C., Local C-semigroups and complete second order abstract Cauchy problems, Stud. Univ. Babeş-Bolyai Math., 61(2016), 211-237.

[17] deLaubenfels, R., Existence Families, Functional Calculi and Evolution Equations, 1570, Lecture Notes in Math., Springer-Verlag, Berlin, 1994.

[18] Li, F., Multiplicative perturbations of incomplete second order abstract differential equations, Kybernetes, 39(2008), 1431-1437.

[19] Li, F., Liang, J., Multiplicative perturbation theorems for regularized cosine functions, Acta Math. Sinica, 46(2003), 119-130.

[20] Li, F., Liu, J., A perturbation theorem for local C-regularized cosine functions, J. Physics: Conference Series, 96(2008), 1-5.

[21] Oka, H., Linear Volterra equation and integrated solution families, Semigroup Forum, 53(1996), 278-297.

[22] Shaw, S.-Y., Li, Y.-C., Characterization and generator of local C-cosine and C-sine functions, Inter. J. Evolution Equations, 1(2005), 373-401.

[23] Takenaka, T., Okazwa, N., A Phillips-Miyadera type perturbation theorem for cosine functions of operators, Tohoku. Math., 69(1990), 257-288.

[24] Takenaka, T., Piskarev, S., Local C-cosine families and $N$-times integrated local cosine families, Taiwanese J. Math., 8(2004), 515-546.

[25] Travis, C.C., Webb, G.F., Perturbation of strongly continuous cosine family generators, Colleq. Math., 45(1981), 277-285.

[26] Wang, S.-W., Gao, M.-C., Automatic extensions of local regularized semigroups and local regularized cosine functions, Proc. Amer. Math. Soc., 127(1999), 1651-1663.

[27] Yeh, N.-S., Kuo, C.-C., Multiplicative perturbations of local $\alpha$-times integrated $C$ semigroups, Acta Math. Sci., 37B(2017), 877-888. 
Chung-Cheng Kuo

Fu Jen Catholic University,

Department of Mathematics,

New Taipei City, Taiwan 24205

e-mail: 033800@fju.edu.tw

Nai-Sher Yeh

Fu Jen Catholic University,

Department of Mathematics,

New Taipei City, Taiwan 24205

e-mail: nyeh@math.fju.edu.tw 